\title{
Secretory Vesicles Targeted to Plasma Membrane During Pollen Germination and Tube Growth
}

\author{
Huaqiang Ruan', Jiang Lit, Ting Wang* and Haiyun Ren* \\ Key Laboratory of Cell Proliferation and Regulation Biology of Ministry of Education, Center for Biological Science and \\ Technology, Advanced Institute of Natural Science, Beijing Normal University, Zhuhai, China
}

\section{OPEN ACCESS}

Edited by:

Junjie $\mathrm{Hu}$,

Institute of Biophysics (CAS), China

Reviewed by:

Eyal Schejter,

Weizmann Institute of Science, Israel Yusong Guo,

Hong Kong University of Science and Technology, Hong Kong

*Correspondence:

Ting wang

twang@bnu.edu.cn

Haiyun Ren

hren@bnu.edu.cn

${ }^{\dagger}$ These authors have contributed equally to this work and share first

authorship

Specialty section:

This article was submitted to Membrane Traffic,

a section of the journal

Frontiers in Cell and Developmental

Biology

Received: 09 October 2020 Accepted: 14 December 2020

Published: 21 January 2021

Citation:

Ruan H, Li J, Wang T and Ren H (2021) Secretory Vesicles Targeted to Plasma Membrane During Pollen

Germination and Tube Growth. Front. Cell Dev. Biol. 8:615447. doi: 10.3389/fcell.2020.615447
Pollen germination and pollen tube growth are important biological events in the sexual reproduction of higher plants, during which a large number of vesicle trafficking and membrane fusion events occur. When secretory vesicles are transported via the F-actin network in proximity to the apex of the pollen tube, the secretory vesicles are tethered and fused to the plasma membrane by tethering factors and SNARE proteins, respectively. The coupling and uncoupling between the vesicle membrane and plasma membrane are also regulated by dynamic cytoskeleton, proteins, and signaling molecules, including small G proteins, calcium, and PIP2. In this review, we focus on the current knowledge regarding secretory vesicle delivery, tethering, and fusion during pollen germination and tube growth and summarize the progress in research on how regulators and signaling molecules participate in the above processes.

Keywords: secretory vesicles, plasma membrane, exocyst complex, SNAREs, regulation, pollen, F-actin

\section{INTRODUCTION}

In seed plants, the production of seeds depends on double fertilization. Mature pollen grains are tri-cellular and composed of two small sperm cells and a large vegetative cell in Arabidopsis. One of the two sperm cells fuses with an egg to form a diploid zygote that develops into an embryo, and the other fuses with the polar nucleus to form a primary endosperm nucleus; this process is called double fertilization (Shi and Yang, 2010). The developmental progression of plant double fertilization is well coordinated: it starts with pollen falling on the stigma; the pollen adheres, hydrates, and germinates on the stigma via specific recognition (Sprunck, 2020). Then, the pollen germinates to produce a tubular structure (the pollen tube) that rapidly elongates through polar growth, penetrates the stigma, and grows in style tissues to ultimately deliver the two immotile sperm cells into the ovule to complete double fertilization (Zheng et al., 2018). Since the proper pollen germination and tube growth are essential for two sperm cells transporting to female gametophyte, so exploring the molecular mechanism of pollen germination and pollen tube growth is of great interest in the field.

Pollen germination and pollen tube elongation form the whole process by which polarity is established and maintained. In this process, many cell wall materials, such as pectins and cellulose, are contained as cargo in vesicles with an average diameter of $0.182 \mu \mathrm{m}$ (Ketelaar et al., 2008; Chebli et al., 2012). These vesicles arise from the Golgi and trans-Golgi network (TGN) and are directionally transported toward and fused with the plasma membrane (PM) at polar exocytosis sites to enable the membrane extension and sustained synthesis of new cell wall material (Wang et al., 2016; Zheng et al., 2018; Grebnev et al., 2020; Guo and Yang, 2020). 
A better understanding of the molecular mechanisms of pollen germination and tube growth is key for successful sexual reproduction.

The process of pollen germination includes polarity establishment and site determination (Liu et al., 2018), after which the germinated pollen can continue membrane expansion for directional tube growth. Via membrane trafficking and integration, cell wall materials, proteins, and other components for germination and membrane expansion are transported and released; these processes are essential for pollen germination and tube growth (Chebli et al., 2012; Wang et al., 2016). It is generally thought that membrane contact between secretory vesicles and the PM is the most important cellular activity that coordinates pollen germination and pollen tube growth ( $\mathrm{Gu}$ and Nielsen, 2013; Guo and Yang, 2020). If directional transportand release-related processes are inhibited, pollen germination and subsequent tube growth and fertilization events will be strongly affected. In recent years, the progress in understanding the dynamic coordination between endocytosis and exocytosis in pollen tubes has been summarized and reviewed (Zhang et al., 2019; Guo and Yang, 2020). In this review, we focus on the process of secretory vesicle directional targeting the PM, which involves vesicle delivery, tethering, and fusion during pollen germination and tip growth, as well as on the different regulators involved, such as some key signaling proteins and other molecules.

\section{VESICLE DELIVERY}

It is generally thought that vesicles are transported by motor protein-mediated directed transport along microfilaments (MFs) toward the target membrane in plant cells, while in animal cells, the microtubule network serves as the track (Coudrier, 2007; Madison et al., 2015; Ueda et al., 2015; Duan and Tominaga, 2018). Experimental data also suggest that plant MFs maintain greater stability than animal MFs and can withstand longdistance vesicle transport (Ren et al., 2019).

Although massive dynamic vesicular transport is dependent on the MF network, the coordination between vesicles and their MF tracks shows quite different dynamic patterns during pollen germination and tube growth (Lan et al., 2018; Liu et al., 2018). Recent research has revealed that actin filaments rotate along the outer edges of pollen grains and then gather in future pollen germination sites, forming collar-like actin structures. Genetic and pharmacological evidence has further revealed an interdependent relationship between the mobility of vesicles and the polymerization of actin filaments. AtFH5, a highly expressed FORMIN protein in Arabidopsis thaliana pollen, is located in vesicles and promotes actin assembly; in turn, the force produced by MF polymerization pushes vesicles to the potential germination site (Figure 1A; Liu et al., 2018). In the pollen tube, the actin cytoskeleton shows a well-organized and highly dynamic structure that might correspond to the specific functions in different regions (Figure 1B; Xiang et al., 2007; Fu, 2015). In the shank region, the parallel F-actin cables are thought to serve as tracks for transporting organelles and vesicles to the pollen tube tip (Hepler and Cheung, 2001; Vidali et al., 2001). In the subapical region, the MFs are short and dense and form a collar-like zone, which might be used as a filter to prevent large organelles and other large membrane structures from entering the tip region (Kroeger et al., 2009; Dong et al., 2012; Diao et al., 2020). In the apical region of the pollen tube, actin filaments are highly dynamic and are thought to organize vesicle docking and fusion with the PM of the pollen tube tip (Vidali et al., 2001; Qu et al., 2013). Interestingly, there exist two alternative possible working patterns between F-actin and secretory vesicles during pollen germination and tube growth (Figures 1A,B). However, it is not yet clear how these two coordination patterns work and whether they equally contribute to pollen germination and tube growth. Furthermore, single molecular techniques and in vitro simulation assays are expected to be introduced that will help to reveal how single F-actin molecules function on vesicles and elucidate the underlying mechanism.

To ensure that secretory vesicles are delivered to the PM along the correct route, actin filaments need to be temporally and spatially coordinated and arranged in a highly dynamic manner. Different classes of actin-binding proteins (ABPs) are involved in this regulation. Among the ABPs, class I formins are very exciting candidate coordinators of actin and vesicle dynamics, since they can localize to secretory vesicles, bind to F-actin, and directly regulate F-actin dynamics (Deeks et al., 2005; Cvrckova et al., 2014; Li S. et al., 2017; Lan et al., 2018; Liu et al., 2018). Lan's et al. research showed that MF nucleation factor formins (FORMIN3 and FORMIN5) can localize to the PM at the tip of the pollen tube and initiate MF assembly (Lan et al., 2018). The pollen germination percentage is significantly reduced in fh3-2 fh5-3 mutant plants. Loss of actin filaments in the pollen tubes of $f h 3 f h 5$ mutants reduces the velocity of tip-directed vesicle transport and alters the apical vesicle accumulation pattern, supporting the idea that apical actin filaments and their regulatory formin proteins can regulate vesicle trafficking (Ye et al., 2009; Lan et al., 2018). It would be very interesting to explore deeply whether and how these pollen-expressed formin proteins are involved in vesicle trafficking and integration processes. Another family of ABPs, the profilins, can interact with formins via the $\mathrm{FH} 1-\mathrm{FH} 2$ domain to enhance filament elongation rates and to thin and elongate actin bundles (Zhang et al., 2016; Li S. et al., 2017). Some actin depolymerization factors, such as ADF5 and actin-bundling proteins (i.e., VILLIN2 and VILLIN5), have been reported to affect actin dynamics, further influencing pollen germination and tube growth; however, there is no evidence showing an interaction with vesicle trafficking or vesicle integration (Qu et al., 2013; Zhu et al., 2017; Diao et al., 2020). Although it is known that the dynamic organization of MFs and their regulatory proteins are essential for targeting of vesicles to the PM for secretion, the underlying molecular mechanism is still unclear, especially regarding how actin organization interacts with secretory vesicles and directs vesicle targeting. Which protein families may be involved during this process still needs to be further explored.

Compared to the role of the MF cytoskeleton in vesicle delivery, the roles of microtubules in pollen tubes are less clear. Pollen tubes contain many microtubule motors of the kinesin family, and pollen-expressed kinesin proteins are believed 


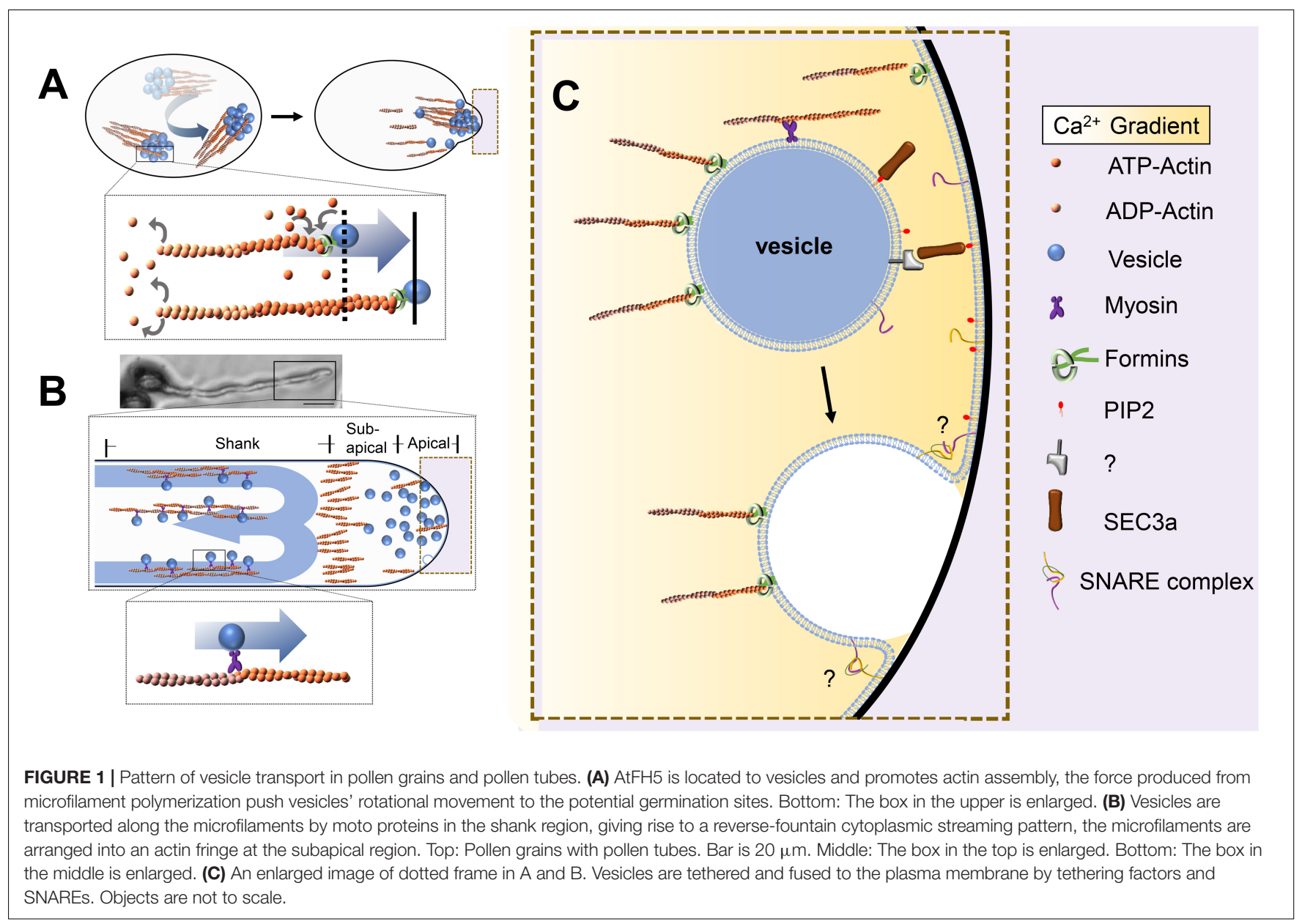

to be involved in the distribution of organelles during pollen tube growth (Cai and Cresti, 2010). Further exploration of the function of the microtubule network, especially the role this network plays in vesicle trafficking during pollen germination and tube growth, would provide more information and help to comprehensively elucidate the role of the cytoskeleton.

Signaling molecules such as the small G protein Rho of plant GTPase (ROP), the second messenger $\mathrm{Ca}^{2+}$, and the phospholipid molecule phosphatidylinositol-4,5-bisphosphate (PIP2) play important roles in pollen germination and vesicle transport in pollen tubes (Berken and Wittinghofer, 2008; Steinhorst and Kudla, 2013; Feiguelman et al., 2018). As molecular switches, small $G$ proteins have two forms: an inactivated GDP-bound form and an active GTP-bound form. ROPs are regulated by ROPGEFs, ROPGAPs, and RHOGDIs (Berken, 2006). In A. thaliana, ROP1, ROP3, and ROP5 are expressed in pollen tubes. ROPs regulate cytoskeletal dynamics and endocytosis through their downstream effector proteins. The pollen-specific protein ROP1 accumulates in the PM of the top of the pollen tube to regulate the $\mathrm{Ca}^{2+}$ concentration gradient, activate the RIC3 pathway, and promote actin depolymerization (Gu et al., 2005; Zhou et al., 2015). Moreover, ROP1 also activates RIC4 to promote actin assembly, change the arrangement of the MF skeleton, and induce the accumulation and transport of vesicles to the tip region ( $\mathrm{Gu}$ et al., 2005; Lee et al., 2008). These results indicate that ROP1 is involved in vesicle transport through regulation of MFs in pollen tubes. However, it is still unclear whether establishment of pollen polarity is also regulated by ROP during pollen germination.

PIP2 localizes at the pollen tube apical PM. The balance of its distribution and content is very important for maintenance of the normal growth of pollen tubes (Monteiro et al., 2005; Zhang and McCormick, 2010). PI(4,5)P2 is synthesized by PIP5K kinase, and the pollen germination and pollen tube polarity growth of the pip5k4 homozygous mutant are significantly impaired (Sousa et al., 2008). Ischebeck et al. (2011) found that overexpression of PIP5K10 or PIP5K11 enlarged the tip of the pollen tube and caused abnormal arrangement of the MF cytoskeleton, indicating that $\mathrm{PI}(4,5) \mathrm{P} 2$ regulates dynamic changes in the MF cytoskeleton. Phosphatidylserine (PS) is abundant in the inverted-cone zone of the apical pollen tube in Arabidopsis. Recent research has revealed that loss of apical localization of PS and significantly decreased distribution lead to obvious decreases in vesicle numbers and an obvious increase in pollen tube width, which indicates that tip-localized PS establishment is important for vesicle targeting/trafficking and polar growth of pollen tubes in Arabidopsis (Zhou et al., 2020). 
Although the oscillation of $\left[\mathrm{Ca}^{2+}\right]_{\text {cyt }}$ follows the growth rate pulse, the oscillation of the pollen tube growth rate is consistent with changes in vesicle dynamics (Holdaway-Clarke et al., 1997; Parton et al., 2001). When pollen tubes reach the growth peak, considerable exocytosis is observed at the top of the test tube. $\mathrm{Ca}^{2+}$-dependent ABP LILIM1 binds F-actin bundles in lily pollen and protects them from depolymerization under low $\left[\mathrm{Ca}^{2+}\right]_{\mathrm{cyt}}$ (Wang et al., 2008). In contrast, with increases in $\left[\mathrm{Ca}^{2+}\right]_{\text {cyt }}$, villin/gelsolin family members cut off actin filaments, decrease the activity of profilin, and reduce the polymerization of MFs (Zhang et al., 2010). The above data indicate that $\mathrm{Ca}^{2+}$ can indirectly affect vesicle transport in pollen tubes. Whether it can also directly affect cytoskeletal dynamics or intracellular vesicle transport remains to be further studied.

\section{VESICLE TETHERING}

After vesicles are delivered in proximity to the target membrane, contact is required between the vesicle and target membrane before fusion, and multisubunit tethering complexes are thought to enable this initial encounter (Figure 1C). The first contact between vesicles and the PM is mediated mainly by the exocyst complex (Yu and Hughson, 2010; Pleskot et al., 2015; Mei et al., 2018). The exocyst complex is composed of the subunits Sec3, Sec5, Sec6, Sec8, Sec10, Sec15, Exo70, and Exo84, which are highly conserved in eukaryotes (TerBush et al., 1996; Mei et al., 2018). There are two models for the mechanism by which the exocyst complex performs its tethering function in yeast and mammalian cells ( $\mathrm{Yu}$ and Hughson, 2010). In the first model, Sec3 and Exo70 interact directly with $\mathrm{P}(4,5) \mathrm{P} 2$ on the PM to mark secretion sites (He et al., 2007; Zhang et al., 2008). The remaining six subunits form a subcomplex, which is recruited to the vesicle membrane through interaction between Sec15 and the Rab GTPase Sec4p (Guo et al., 1999). In the other model, all eight subunits of the exocyst complex assemble into two different subcomplexes. One of them is composed of Sec3, Sec8, Sec5, and Sec6, which are anchored to the membrane through Sec3 and directly interact with $\mathrm{P}(4,5) \mathrm{P} 2$. Another subcomplex consists of Sec10, Sec15, Exo70, and Exo84, which are located on the vesicle. Then, interaction between Sec8 and Sec10 assembles the two subcomplexes into a complete exocyst complex to complete the process of vesicle tethering to the PM (Katoh et al., 2015; Heider et al., 2016; Polgar and Fogelgren, 2018). However, the molecular mechanism of the exocyst complex in plants is still poorly understood. Mutations in plant exocyst subunits, such as sec6, sec $15 a$, and $\sec 5 a / \sec 5 b$ single and double mutations, cause defects in pollen germination and tube growth, while $\sec 8$ and $\sec 3 a$ mutations have been reported to cause malespecific transmission defects (Cole et al., 2005; Bloch et al., 2016; Li Y. et al., 2017).

Pollen grain germination has been found to be defective in a $\sec 3 a / S E C 3 A$ heterozygous mutant (Li Y. et al., 2017), while in overexpression lines, multiple tips emerge from pollen grain surfaces, and GFP-SEC3A signals appear only in the PM at the tip of the growing pollen tube (Bloch et al., 2016). These results suggest that SEC3A plays an important role in establishing polarity during pollen germination and tube growth. In addition, in sec3a/GFP-SEC3A-overexpressing complementation lines, a strong positive correlation between the localization of GFPSEC3A at the tips of growing pollen tubes and the secretion of esterified pectins suggests that GFP-SEC3A might work as an intracellular marker for exocytosis. It would be very interesting to explore how SEC3A and other components coordinate to participate in the vesicle secretion process and which other essential proteins/other molecules are secreted by the exocystrelated pathway. The localization and dynamics of SEC8 in pollen tubes are consistent with those of SEC3A, and the homozygous sec8-null mutant also shows defects in male-specific transmission, similar to the sec3a mutant (Cole et al., 2005; Hala et al., 2008), which indicates that SEC3A and SEC8 may function together to participate in polar transport and the release of key contents for germination and tube growth. There are 23 potential EXO70 genes in Arabidopsis, and mutations in EXO70 subunits cause different defects in pollen germination and tube growth (Elias, 2003; Synek et al., 2006, 2017; Li et al., 2010; Vukasinovic and Zarsky, 2016). The diversity of EXO70 family genes implies that there is a large degree of functional redundancy among the subunits. EXO70C2 seems to play a dominant role together with EXO70C1, since the exo70c1exo70c2 double mutation causes a complete pollen-specific transmission defect (Synek et al., 2017). The yeast two-hybrid system has identified interactions of Arabidopsis SEC3A with EXO70A1, SEC10 with SEC15b, and SEC6 with SEC8; these findings indicate that the plant exocyst complex might be structurally conserved and similar to that in animal and yeast cells (Hala et al., 2008). Given data from dynamic imaging analysis of exocyst components in other plant tissues and cells (e.g., root epidermal cells) and the molecular mechanisms of tethering in other cell types (Fendrych et al., 2013), it is reasonable to hypothesize that the exocyst complex also functions as a tethering complex for vesicle transport during pollen germination and tube growth. In the future, some alternative imaging methods could be developed and applied to pollen cells to track the dynamic pattern of each particle component. The results of such studies would offer us more details about how the exocyst complex functions in tethering secretory vesicles.

In yeast cells, sec3p and Exo70p can be recruited to the $\mathrm{PM}$ by binding directly with $\mathrm{P}(4,5) \mathrm{P} 2$, and other subunits are assembled at the active secretion site by Sec4p (Novick et al., 2006). Similarly, the exocyst complex is also expressed in polar secretory active sites in animal cells (Anitei et al., 2006). Although the interaction of SEC3A and PIP2 in plant cells has been confirmed by in vitro assays, in vivo analysis of the truncated SEC3A protein with loss of the key PIP2 interaction domain has indicated that the interaction does not affect SEC3A apical PM localization and functionality in Arabidopsis (Bloch et al., 2016). However, different results from ectopic expression of the same truncated protein have been obtained in tobacco cells, so the interaction and functionality between PIP2 and exocyst components in plant cells still need to be explored (Bloch et al., 2016; Li Y. et al., 2017).

The Rho small G proteins Cdc42, RHO1, and RHO3 regulate the polar localization of $\sec 3 \mathrm{p}$ and exo70p in yeast cells 
(Finger et al., 1998; Zhang et al., 2008). In plant cells, it has been found that ICR/RIP, an effector protein of ROP, can form a complex with active ROP1 and SEC3A to regulate root cell polar growth (Lavy et al., 2007; Li Y. et al., 2017; Li et al., 2008). It would be very interesting to screen and identify whether some ROP effector or regulatory proteins interact with the exocyst complex and coordinate the tethering process during pollen germination and tube growth.

A general model of exocyst action suggests that most of the components that arrive at the PM and tether secretory vesicles cannot localize properly after disruption of the actin cytoskeleton (Synek et al., 2014). In budding yeast, gene mutations in SEC10 and SEC15 strongly affect the cytoskeleton, leading to significant defects in the actin cytoskeleton (Aronov and Gerst, 2004). Inhibition of the interaction between EXO70 and the Arp2/3 complex blocks the formation of actin-based membrane protrusions and affects cell motility in animal cells, which indicates the special role EXO70 might play in coordinating the cytoskeleton and membrane trafficking during cell migration (Zuo et al., 2006). Recent research has revealed that For1F is a fusion protein containing both the exocyst complex subunit (SEC10) domain and the conserved actin-nucleating factor (formin) domain and that this new fusion protein is essential for polar growth in Physcomitrella patens (van Gisbergen et al., 2018). This work suggests that both the exocyst complex and actin filaments are essential and cooperate in tethering secretory vesicles during exocytosis. Further exploration of exocystcytoskeleton interactions in different cell types would offer some very important clues and elucidate possible cooperative strategies in plant cells, which could help us to better understand the molecular mechanism of vesicle trafficking.

\section{VESICLE FUSION}

Membrane fusion occurs after the tethering of vesicles and target membranes; soluble $N$-ethylmaleimide-sensitive factor attachment receptors (SNAREs) play a major role in membrane fusion Figure 1C (Uemura et al., 2004; Lipka et al., 2007; Sanderfoot, 2007). SNAREs are classified as Qa-, Qb-, Qc-, and R-SNAREs based on their conserved residues, and all contain a hydrophobic SNARE domain. The SNARE proteins located in the PM and endosomes are listed in Figure 2. The Qa-SNARE family members AtSYP124, AtSYP125, and AtSYP131 are exclusively expressed in male gametophytes (Silva et al., 2010; Ichikawa et al., 2015; Slane et al., 2017). The syp124syp125syp131 mutant shows more severe male gametophyte defects than the syp 124 syp 125 double mutant, and the pollen tube stops growing during passage through the style, suggesting functional redundancy (Silva et al., 2010; Ichikawa et al., 2015; Slane et al., 2017). SYP131 is mainly stably located in the PM, while SYP124/SYP125 seems to circulate between the PM and endosomes. Therefore, SYP124 and SYP125 may be responsible for membrane fusion in the recycling pathway, while SYP131 may preferentially mediate the membrane fusion of secretory vesicles and contribute to the growth of pollen tubes (Silva et al., 2010; Ichikawa et al., 2015; Slane et al., 2017). VAMP72 family proteins are plant-specific

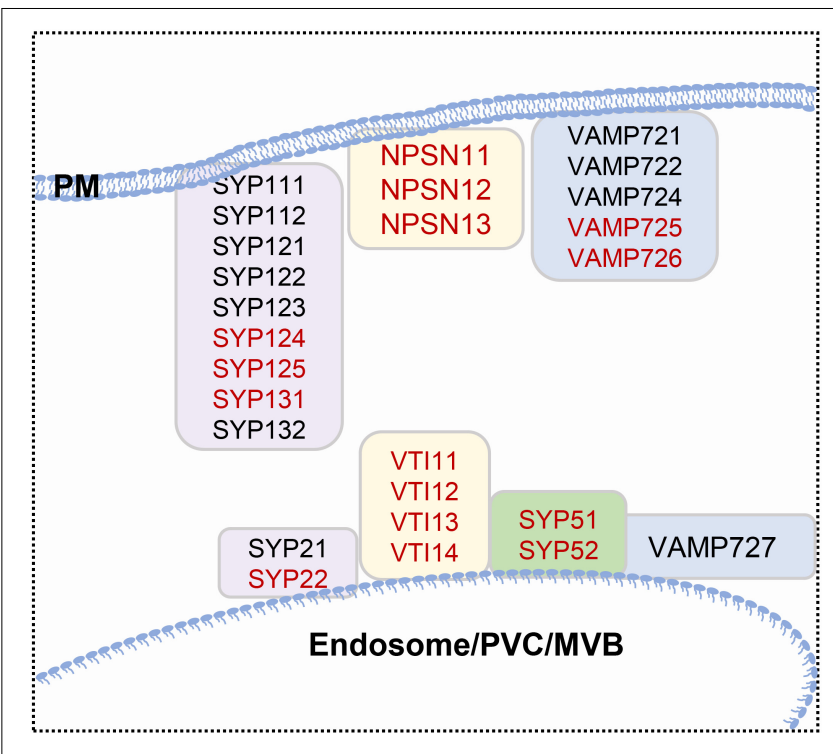

\section{Qa-SNAREs Qb-SNAREs Qc-SNAREs R-SNAREs}

FIGURE 2 | SNAREs located in the plasma membrane and endosomes. Genes that are highly expressed in pollen, which are highlighted in red.

R-SNARE proteins that are located mainly in the PM (Lipka et al., 2007). It has been found that the pollen tubes of vamp $721^{+/-}$ vamp $722^{+/-}$show a certain proportion of curly phenotypes, and half of them lack the $\mathrm{Ca}^{2+}$ channel AtCNGC18 on the PM (Meng et al., 2020). The N-terminal longin domains of AtVAMP721 and AtVAMP722 interact with AtMLO5, recruit AtCNGC18 to relocate to the PM, affect the local cytoplasmic $\mathrm{Ca}^{2+}$ concentration, and regulate the directional responses of pollen tubes to extracellular signals (Meng et al., 2020). However, research on SNAREs has been scarce; thus far, there have been no reports about $\mathrm{Qb}$ - and Qc-SNARE proteins in pollen. The specific members of the SNARE complex that are expressed in pollen and their functions in pollen germination and tube growth are unclear.

Many studies have shown that $\mathrm{Ca}^{2+}$ plays an important regulatory role in vesicle fusion (Konopka-Postupolska and Clark, 2017). In Arabidopsis, the localization of Qa-SNARE in pollen is also regulated by $\mathrm{Ca}^{2+}$ ions. The polarity establishment of SYP125 before germination seems to be related to the establishment of a $\mathrm{Ca}^{2+}$ gradient, and the location of SYP124 and SYP125 is also changed while $\mathrm{Ca}^{2+}$ flux is disturbed, suggesting that $\mathrm{Ca}^{2+}$ regulates vesicle fusion in many ways (Silva et al., 2010; Ichikawa et al., 2015). In addition, the distribution of SYP124 and SYP125 is closely related to MFs, MFs depolymerization destroys their localization (Silva et al., 2010; Ichikawa et al., 2015); and some actin or actin-related proteins have been identified in a interactome analysis of SNARE proteins (Fujiwara et al., 2014). In animal cells, synaptotagmin (SYT) proteins have been reported to regulate vesicle fusion (Kweon et al., 2019). There are seven SYTs in Arabidopsis (Ishikawa et al., 2020). In the plant SYT family, SYT1 is the 
most extensively characterized protein; it acts as an endoplasmic reticulum (ER)-PM tethering factor and participates in biotic and abiotic stress responses in plants (Schapire et al., 2008; Siao et al., 2016). SYTs contain conserved C-terminal tandem C2A and C2B domains and interact with phosphatidylinositol, SNAREs, and $\mathrm{Ca}^{2+}$ channel proteins to regulate endocytosis/exocytosis ( $\mathrm{Wu}$ et al., 2014). It will be interesting to study whether plant cells implement a similar regulatory mechanism in the processes of pollen germination and tube growth. SYT2 is expressed mainly in Arabidopsis pollen and is located in the Golgi and PM, and the SYT2-C2AB domain binds to the phospholipid membrane in a $\mathrm{Ca}^{2+}$-dependent manner (Wang et al., 2015). The pollen germination rate of syt 2 mutants is decreased, and pollen tube elongation is restricted (Wang et al., 2015), but the relationship between SYT2 and vesicle fusion during pollen germination and pollen tube growth needs to be further confirmed. Annexin, a $\mathrm{Ca}^{2+}$ channel protein, can bind to membrane phospholipids in a $\mathrm{Ca}^{2+}$-dependent manner and can also bind MFs. Thus, it may provide an important connection among intracellular $\mathrm{Ca}^{2+}$ signaling, the actin cytoskeleton, and the membrane and participate in intracellular vesicle trafficking (KonopkaPostupolska and Clark, 2017). Ann5 is a $\mathrm{Ca}^{2+}$ channel protein that is highly expressed in mature pollen grains and pollen tubes of A. thaliana, and a decrease in its expression leads to severe sterility (Lichocka et al., 2018). Ann5 seems to participate in pollen development, germination, and pollen tube elongation by promoting $\mathrm{Ca}^{2+}$-regulated intimal transport, but the exact mechanism needs to be further studied (Zhu et al., 2014a,b; Lichocka et al., 2018).

\section{PROSPECTS}

Pollen germination and pollen tube growth are important biological processes in plant sexual reproduction. Many vesicle trafficking, tethering, and fusion events take place during polar pollen germination and tube elongation. In past years, some key tethered factors and SNARE family members have been

\section{REFERENCES}

Anitei, M., Ifrim, M., Ewart, M. A., Cowan, A. E., Carson, J. H., Bansal, R., et al. (2006). A role for Sec8 in oligodendrocyte morphological differentiation. J. Cell Sci. 119, 807-818. doi: 10.1242/jcs.02785

Aronov, S., and Gerst, J. E. (2004). Involvement of the late secretory pathway in actin regulation and mRNA transport in yeast. J. Biol. Chem. 279, 36962-36971. doi: $10.1074 /$ jbc.M402068200

Berken, A. (2006). ROPs in the spotlight of plant signal transduction. Cell Mol. Life Sci. 63, 2446-2459. doi: 10.1007/s00018-006-6197-1

Berken, A., and Wittinghofer, A. (2008). Structure and function of Rho-type molecular switches in plants. Plant Physiol. Biochem. 46, 380-393. doi: 10.1016/ j.plaphy.2007.12.008

Bloch, D., Pleskot, R., Pejchar, P., Potocky, M., Trpkosova, P., Cwiklik, L., et al. (2016). Exocyst SEC3 and phosphoinositides define sites of exocytosis in pollen tube initiation and growth. Plant Physiol. 172, 980-1002. doi: 10.1104/pp.16. 00690

Cai, G., and Cresti, M. (2010). Microtubule motors and pollen tube growth-still an open question. Protoplasma 247, 131-143. doi: 10.1007/s00709-010-0214-9

Chebli, Y., Kaneda, M., Zerzour, R., and Geitmann, A. (2012). The cell wall of the Arabidopsis pollen tube-spatial distribution, recycling, and network formation of polysaccharides. Plant Physiol. 160, 1940-1955. doi: 10.1104/pp.112.199729 identified and characterized. However, there are still some key issues that have not yet been resolved, such as the molecular mechanism of each subunit of the exocyst complex and each member of the SNARE family during pollen germination and pollen tube growth. Recent studies have revealed that actin filaments not only participate in intracellular vesicle transport as tracks but also provide the driving forces for vesicle trafficking. Further study is needed to determine whether and how MFs function in vesicle tethering and fusion with the PM and to reveal the interplay between these processes. In addition, $\mathrm{Ca}^{2+}$ is an important signaling molecule for pollen germination and tube growth (Iwano et al., 2004; Hepler et al., 2012; Steinhorst and Kudla, 2013); thus, it will be very meaningful to study which and how $\mathrm{Ca}^{2+}$ channels or calcium binding proteins are involved in the regulation of vesicle fusion during pollen germination and pollen tube growth. It is believed that the development of microscopic technologies and research methods will enable in-depth analysis of vesicle delivery, tethering, and fusion to the PM during pollen germination and pollen tube growth.

\section{AUTHOR CONTRIBUTIONS}

HR drew the figure and wrote the third part "vesicle fusion." JL wrote the first and second part of the manuscripts "vesicle delivery" and "vesicle tethering." TW wrote and revised the manuscript. HR designed and organized the concept of the manuscript and revised the manuscript. All authors contributed to the article and approved the submitted version.

\section{ACKNOWLEDGMENTS}

We are grateful for financial support from the National Natural Science Research Foundation of China (91854206 and 31770206 to HR).

Cole, R. A., Synek, L., Zarsky, V., and Fowler, J. E. (2005). SEC8, a subunit of the putative Arabidopsis exocyst complex, facilitates pollen germination and competitive pollen tube growth. Plant Physiol. 138, 2005-2018. doi: 10.1104/ pp.105.062273

Coudrier, E. (2007). Myosins in melanocytes: to move or not to move? Pigment Cell Res. 20, 153-160. doi: 10.1111/j.1600-0749.2007.00376.x

Cvrckova, F., Oulehlova, D., and Zarsky, V. (2014). Formins: linking cytoskeleton and endomembranes in plant cells. Int. J. Mol. Sci. 16, 1-18. doi: 10.3390/ ijms 16010001

Deeks, M. J., Cvrckova, F., Machesky, L. M., Mikitova, V., Ketelaar, T., Zarsky, V., et al. (2005). Arabidopsis group le formins localize to specific cell membrane domains, interact with actin-binding proteins and cause defects in cell expansion upon aberrant expression. New Phytol. 168, 529-540. doi: 10.1111/ j.1469-8137.2005.01582.x

Diao, M., Li, X., and Huang, S. (2020). Arabidopsis AIP1-1 regulates the organization of apical actin filaments by promoting their turnover in pollen tubes. Sci. China Life Sci. 63, 239-250. doi: 10.1007/s11427-019-9532-0

Dong, H., Pei, W., and an Ren, H. (2012). Actin fringe is correlated with tip growth velocity of pollen tubes. Mol. Plant 5, 1160-1162. doi: 10.1093/mp/sss073

Duan, Z., and Tominaga, M. (2018). Actin-myosin XI: an intracellular control network in plants. Biochem. Biophys. Res. Commun. 506, 403-408. doi: 10.1016/ j.bbrc.2017.12.169 
Elias, M. (2003). The exocyst complex in plants. Cell Biol. Int. 27, 199-201. doi: 10.1016/s1065-6995(02)00349-9

Feiguelman, G., Fu, Y., and Yalovsky, S. (2018). ROP GTPases structure-function and signaling pathways. Plant Physiol. 176, 57-79. doi: 10.1104/pp.17.01415

Fendrych, M., Synek, L., Pecenkova, T., Drdova, E. J., Sekeres, J., de Rycke, R., et al. (2013). Visualization of the exocyst complex dynamics at the plasma membrane of Arabidopsis thaliana. Mol. Biol. Cell. 24, 510-520. doi: 10.1091/mbc.E12-060492

Finger, F. P., Hughes, T. E., and Novick, P. (1998). Sec3p is a spatial landmark for polarized secretion in budding yeast. Cell 92, 559-571.

$\mathrm{Fu}$, Y. (2015). The cytoskeleton in the pollen tube. Curr. Opin. Plant Biol. 28, 111-119. doi: 10.1016/j.pbi.2015.10.004

Fujiwara, M., Uemura, T., Ebine, K., Nishimori, Y., Ueda, T., Nakano, A., et al. (2014). Interactomics of Qa-SNARE in Arabidopsis thaliana. Plant Cell Physiol. 55, 781-789. doi: 10.1093/pcp/pcu038

Grebnev, G., Cvitkovic, M., Fritz, C., Cai, G., Smith, A. S., and Kost, B. (2020). Quantitative structural organization of bulk apical membrane traffic in pollen tubes. Plant Physiol. 183, 1559-1585.

Gu, F., and Nielsen, E. (2013). Targeting and regulation of cell wall synthesis during tip growth in plants. J. Integr. Plant Biol. 55, 835-846. doi: 10.1111/jipb.12077

Gu, Y., Fu, Y., Dowd, P., Li, S., Vernoud, V., Gilroy, S., et al. (2005). A Rho family GTPase controls actin dynamics and tip growth via two counteracting downstream pathways in pollen tubes. J. Cell Biol. 169, 127-138. doi: 10.1083/ jcb. 200409140

Guo, J., and Yang, Z. (2020). Exocytosis and endocytosis: coordinating and finetuning the polar tip growth domain in pollen tubes. J. Exp. Bot. 71, 2428-2438. doi: $10.1093 / \mathrm{jxb} / \mathrm{eraa} 134$

Guo, W., Roth, D., Walch-Solimena, C., and Novick, P. (1999). The exocyst is an effector for Sec4p, targeting secretory vesicles to sites of exocytosis. The EMBO J. 18, 1071-1080.

Hala, M., Cole, R., Synek, L., Drdova, E., Pecenkova, T., Nordheim, A., et al. (2008). An exocyst complex functions in plant cell growth in Arabidopsis and tobacco. Plant Cell 20, 1330-1345. doi: 10.1105/tpc.108.059105

He, B., Xi, F., Zhang, J., TerBush, D., Zhang, X., and Guo, W. (2007). Exo70p mediates the secretion of specific exocytic vesicles at early stages of the cell cycle for polarized cell growth. J. Cell Biol. 176, 771-777.

Heider, M. R., Gu, M., Duffy, C. M., Mirza, A. M., Marcotte, L. L., Walls, A. C., et al. (2016). Subunit connectivity, assembly determinants and architecture of the yeast exocyst complex. Nat. Struct. Mol. Biol. 23, 59-66.

Hepler, P. K., and Cheung, A. (2001). Polarized cell growth in higher plants. Annu. Rev. Cell Dev. Bio. 17, 159-187.

Hepler, P. K., Kunkel, J. G., Rounds, C. M., and Winship, L. J. (2012). Calcium entry into pollen tubes. Trends Plant Sci. 17, 32-38. doi: 10.1016/j.tplants.2011.10.007

Holdaway-Clarke, T. L., Feijo, J. A., Hackett, G. R., Kunkel, J. G., and Hepler, P. K. (1997). Pollen tube growth and the intracellular cytosolic calcium gradient oscillate in phase while extracellular calcium influx is delayed. Plant Cell. 9, 1999-2010. doi: 10.1105/tpc.9.11.1999

Ichikawa, M., Iwano, M., and Sato, M. H. (2015). Nuclear membrane localization during pollen development and apex-focused polarity establishment of SYP124/125 during pollen germination in Arabidopsis thaliana. Plant Reprod. 28, 143-151. doi: 10.1007/s00497-015-0265-3

Ischebeck, T., Stenzel, I., Hempel, F., Jin, X., Mosblech, A., and Heilmann, I. (2011). Phosphatidylinositol-4,5-bisphosphate influences Nt-Rac5-mediated cell expansion in pollen tubes of Nicotiana tabacum. Plant J. 65, 453-468. doi: 10.1111/j.1365-313X.2010.04435.x

Ishikawa, K., Tamura, K., Fukao, Y., and Shimada, T. (2020). Structural and functional relationships between plasmodesmata and plant endoplasmic reticulum-plasma membrane contact sites consisting of three synaptotagmins. New Phytol. 226, 798-808.

Iwano, M., Shiba, H., Miwa, T., Che, F. S., Takayama, S., Nagai, T., et al. (2004). $\mathrm{Ca}^{2+}$ dynamics in a pollen grain and papilla cell during pollination of Arabidopsis. Plant Physiol. 136, 3562-3571. doi: 10.1104/pp.104.046961

Katoh, Y., Nozaki, S., Hartanto, D., Miyano, R., and Nakayama, K. (2015). Architectures of multisubunit complexes revealed by a visible immunoprecipitation assay using fluorescent fusion proteins. J. Cell Sci. 128, 2351-2362.

Ketelaar, T., Galway, M. E., Mulder, B. M., and Emons, A. M. (2008). Rates of exocytosis and endocytosis in Arabidopsis root hairs and pollen tubes. J. Microscopy 231, 265-273.
Konopka-Postupolska, D., and Clark, G. (2017). Annexins as overlooked regulators of membrane trafficking in plant cells. Int. J. Mol. Sci. 18:863. doi: 10.3390/ ijms 18040863

Kroeger, J. H., Daher, F. B., Grant, M., and Geitmann, A. (2009). Microfilament orientation constrains vesicle flow and spatial distribution in growing pollen tubes. Biophys. J. 97, 1822-1831. doi: 10.1016/j.bpj.2009.07.038

Kweon, D. H., Kong, B., and Shin, Y. K. (2019). Search for a minimal machinery for $\mathrm{Ca}^{2+}$-triggered millisecond neuroexocytosis. Neuroscience 420, 4-11. doi: 10.1016/j.neuroscience.2018.07.031

Lan, Y., Liu, X., Fu, Y., and Huang, S. (2018). Arabidopsis class I formins control membrane-originated actin polymerization at pollen tube tips. PLoS Genet. 14:e1007789. doi: 10.1371/journal.pgen.1007789

Lavy, M., Bloch, D., Hazak, O., Gutman, I., Poraty, L., Sorek, N., et al. (2007). A novel ROP/RAC effector links cell polarity, root-meristem maintenance, and vesicle trafficking. Cur. Biol. 17, 947-952. doi: 10.1016/j.cub.2007.04.038

Lee, Y. J., Szumlanski, A., Nielsen, E., and Yang, Z. (2008). Rho-GTPase-dependent filamentous actin dynamics coordinate vesicle targeting and exocytosis during tip growth. J. Cell Biol. 181, 1155-1168. doi: 10.1083/jcb.200801086

Li, S., Dong, H., Pei, W., Liu, C., Zhang, S., Sun, T., et al. (2017). LlFH1-mediated interaction between actin fringe and exocytic vesicles is involved in pollen tube tip growth. New Phytol. 214, 745-761. doi: 10.1111/nph.14395

Li, Y., Tan, X., Wang, M., Li, B., Zhao, Y., Wu, C., et al. (2017). Exocyst subunit SEC3A marks the germination site and is essential for pollen germination in Arabidopsis thaliana. Sci. Rep. 7:40279. doi: 10.1038/srep40279

Li, S., Gu, Y., Yan, A., Lord, E., and Yang, Z. (2008). RIP1 (ROP Interactive Partner 1)/ICR1 marks pollen germination sites and may act in the ROP1 pathway in the control of polarized pollen growth. Mol. Plant 1, 1021-1035. doi: $10.1093 / \mathrm{mp} / \mathrm{ssn} 051$

Li, S., van Os, G. M., Ren, S., Yu, D., Ketelaar, T., Emons, A. M., et al. (2010). Expression and functional analyses of EXO70 genes in Arabidopsis implicate their roles in regulating cell type-specific exocytosis. Plant Physiol. 154, 18191830. doi: $10.1104 /$ pp. 110.164178

Lichocka, M., Rymaszewski, W., Morgiewicz, K., Barymow-Filoniuk, I., Chlebowski, A., Sobczak, M., et al. (2018). Nucleus- and plastid-targeted annexin 5 promotes reproductive development in Arabidopsis and is essential for pollen and embryo formation. BMC Plant Biol. 18:183. doi: 10.1186/s12870-018-1405-3

Lipka, V., Kwon, C., and Panstruga, R. (2007). SNARE-ware: the role of SNAREdomain proteins in plant biology. Annu. Rev. Cell Dev. Biol. 23, 147-174. doi: 10.1146/annurev.cellbio.23.090506.123529

Liu, C., Zhang, Y., and Ren, H. (2018). Actin polymerization mediated by AtFH5 directs the polarity establishment and vesicle trafficking for pollen germination in Arabidopsis. Mol. Plant. 11, 1389-1399. doi: 10.1016/j.molp.2018.09.004

Madison, S. L., Buchanan, M. L., Glass, J. D., McClain, T. F., Park, E., and Nebenfuhr, A. (2015). Class XI myosins move specific organelles in pollen tubes and are required for normal fertility and pollen tube growth in arabidopsis. Plant Physiol. 169, 1946-1960. doi: 10.1104/pp.15.01161

Mei, K., Li, Y., Wang, S., Shao, G., Wang, J., Ding, Y., et al. (2018). Cryo-EM structure of the exocyst complex. Nat. Struct. Mol. Biol. 25, 139-146. doi: 10. 1038/s41594-017-0016-2

Meng, J., Liang, L., Jia, P., Wang, Y., Li, H., and Yang, W. (2020). Integration of ovular signals and exocytosis of a $\mathrm{Ca}^{2+}$ channel by MLOs in pollen tube guidance. Nat. Plants. 6, 143-153. doi: 10.1038/s41477-0200599-1

Monteiro, D., Liu, Q., Lisboa, S., Scherer, G. E., Quader, H., and Malho, R. (2005). Phosphoinositides and phosphatidic acid regulate pollen tube growth and reorientation through modulation of $\left[\mathrm{Ca}^{2+}\right] \mathrm{c}$ and membrane secretion. J. Exp. Bot. 56, 1665-1674. doi: 10.1093/jxb/eri163

Novick, P., Medkova, M., Dong, G., Hutagalung, A., Reinisch, K., and Grosshans, B. (2006). Interactions between Rabs, tethers, SNAREs and their regulators in exocytosis. Biochem. Soc. Trans. 34, 683-686. doi: 10.1042/BST0340683

Parton, R. M., Fischer-Parton, S., Watahiki, M. K., and Trewavas, A. J. (2001). Dynamics of the apical vesicle accumulation and the rate of growth are related in individual pollen tubes. J. Cell Sci. 114, 2685-2695.

Pleskot, R., Cwiklik, L., Jungwirth, P., Zarsky, V., and Potocky, M. (2015). Membrane targeting of the yeast exocyst complex. Biochim Biophys. Acta 1848, 1481-1489. doi: 10.1016/j.bbamem.2015.03.026

Polgar, N., and Fogelgren, B. (2018). Regulation of cell polarity by exocyst-mediated trafficking. Cold Spring Harb. Perspect. Biol. 10:a031401. 
Qu, X., Zhang, H., Xie, Y., Wang, J., Chen, N., and Huang, S. (2013). Arabidopsis villins promote actin turnover at pollen tube tips and facilitate the construction of actin collars. Plant Cell 25, 1803-1817. doi: 10.1105/tpc.113.110940

Ren, Z., Zhang, Y., Zhang, Y., He, Y., Du, P., Wang, Z., et al. (2019). Cryo-EM structure of actin filaments from zea mays pollen. Plant Cell 31, 2855-2867. doi: $10.1105 /$ tpc.18.00973

Sanderfoot, A. (2007). Increases in the number of SNARE genes parallels the rise of multicellularity among the green plants. Plant Physiol. 144, 6-17. doi: 10.1104/pp.106.092973

Schapire, A. L., Voigt, B., Jasik, J., Rosado, A., Lopez-Cobollo, R., Menzel, D., et al. (2008). Arabidopsis synaptotagmin 1 is required for the maintenance of plasma membrane integrity and cell viability. Plant Cell 12, 3374-3388. doi: $10.1105 /$ tpc. 108.063859

Shi, D. Q., and Yang, W. C. (2010). Pollen germination and tube growth. Plant Dev. Biol. Biotechnol. Perspect. 1, 245-282.

Siao, W., Wang, P., Voigt, B., Hussey, P. J., and Baluska, F. (2016). Arabidopsis SYT1 maintains stability of cortical endoplasmic reticulum networks and VAP27-1-enriched endoplasmic reticulum-plasma membrane contact sites. J. Exp. Bot. 67, 6161-6171. doi: 10.1093/jxb/erw381

Silva, P. A., Ul-Rehman, R., Rato, C., Sansebastiano, G. P. D., and Malho, R. (2010). Asymmetric localization of Arabidopsis SYP124 syntaxin at the pollen tube apical and sub-apical zones is involved in tip growth. BMC Plant Biol. 10:179. doi: 10.1186/1471-2229-10-179

Slane, D., Reichardt, I., El Kasmi, F., Bayer, M., and Jurgens, G. (2017). Evolutionarily diverse SYP1 Qa-SNAREs jointly sustain pollen tube growth in Arabidopsis. Plant J. 92, 375-385. doi: 10.1111/tpj.13659

Sousa, E., Kost, B., and Malho, R. (2008). Arabidopsis phosphatidylinositol-4monophosphate 5-kinase 4 regulates pollen tube growth and polarity by modulating membrane recycling. Plant Cell 20, 3050-3064. doi: 10.1105/tpc. 108.058826

Sprunck, S. (2020). Twice the fun, double the trouble: gamete interactions in flowering plants. Curr. Opin. Plant Biol. 53, 106-116. doi: 10.1016/j.pbi.2019. 11.003

Steinhorst, L., and Kudla, J. (2013). Calcium - a central regulator of pollen germination and tube growth. Biochim Biophys Acta. 1833, 1573-1581. doi: 10.1016/j.bbamcr.2012.10.009

Synek, L., Schlager, N., Elias, M., Quentin, M., Hauser, M. T., and Zarsky, V. (2006). AtEXO70A1, a member of a family of putative exocyst subunits specifically expanded in land plants, is important for polar growth and plant development. Plant J. 48, 54-72. doi: 10.1111/j.1365-313X.2006.02854.x

Synek, L., Sekeres, J., and Zarsky, V. (2014). The exocyst at the interface between cytoskeleton and membranes in eukaryotic cells. Front. Plant Sci. 4:543. doi: 10.3389/fpls.2013.00543

Synek, L., Vukasinovic, N., Kulich, I., Hala, M., Aldorfova, K., Fendrych, M., et al. (2017). EXO70C2 is a key regulatory factor for optimal tip growth of pollen. Plant Physiol. 174, 223-240. doi: 10.1104/pp.16.01282

TerBush, D. R., Maurice, T., Roth, D., and Novick, P. (1996). The Exocyst is a multiprotein complex required for exocytosis in Saccharomyces cerevisiae. The EMBO Journal 15, 6483-6494. doi: 10.1002/j.1460-2075.1996.tb01039.x

Ueda, H., Tamura, K., and Hara-Nishimura, I. (2015). Functions of plant-specific myosin XI: from intracellular motility to plant postures. Curr. Opin. Plant Biol. 28, 30-38. doi: 10.1016/j.pbi.2015.08.006

Uemura, T., Ueda, T., Ohniwa, R. L., Nakano, A., Takeyasu, K., and Sato, M. H. (2004). Systematic analysis of SNARE molecules in Arabidopsis: dissection of the post-Golgi network in plant cells. Cell Struct. Funct. 29, 49-65. doi: 10.1247/ csf.29.49

van Gisbergen, P. A. C., Wu, S., Chang, M., Pattavina, K. A., Bartlett, M. E., and Bezanilla, M. (2018). An ancient Sec10-formin fusion provides insights into actin-mediated regulation of exocytosis. J. Cell Biol. 217, 945-957. doi: $10.1083 /$ jcb.201705084

Vidali, L., McKenna, S. T., and Hepler, P. K. (2001). Actin polymerization is essential for pollen tube growth. Mol. Biol. Cell 12, 2534-2545. doi: 10.1091/ mbc.12.8.2534

Vukasinovic, N., and Zarsky, V. (2016). Tethering complexes in the Arabidopsis endomembrane system. Front. Cell Dev. Biol. 4:46. doi: 10.3389/fcell.2016. 00046

Wang, H., Han, S., Siao, W., Song, C., Xiang, Y., Wu, X., et al. (2015). Arabidopsis synaptotagmin 2 participates in pollen germination and tube growth and is delivered to plasma membrane via conventional secretion. Mol. Plant 8, 1737-1750. doi: 10.1016/j.molp.2015.09.003

Wang, H., Wan, A., and Jauh, G. Y. (2008). An actin-binding protein, LlLIM1, mediates calcium and hydrogen regulation of actin dynamics in pollen tubes. Plant Physiol. 147, 1619-1636. doi: 10.1104/pp.108.118604

Wang, H., Zhuang, X., Wang, X., Law, A. H., Zhao, T., Du, S., et al. (2016). A Distinct pathway for polar exocytosis in plant cell wall formation. Plant Physiol. 172, 1003-1018. doi: 10.1104/pp.16.00754

Wu, L., Hamid, E., Shin, W., and Chiang, H. (2014). Exocytosis and endocytosis: modes, functions, and coupling mechanisms. Annu. Rev. Physiol. 76, 301-331. doi: 10.1146/annurev-physiol-021113-170305

Xiang, Y., Huang, X., Wang, T., Zhang, Y., Liu, Q., Hussey, P. J., et al. (2007). Actin binding protein 29 from lilium pollen plays an important role in dynamic actin remodeling. Plant Cell 19, 1930-1946. doi: 10.1105/tpc.106.048413

Ye, J., Zheng, Y., Yan, A., Chen, N., Wang, Z., Huang, S., et al. (2009). Arabidopsis formin3 directs the formation of actin cables and polarized growth in pollen tubes. Plant Cell 21, 3868-3884. doi: 10.1105/tpc.109.068700

Yu, I. M., and Hughson, F. M. (2010). Tethering factors as organizers of intracellular vesicular traffic. Annu. Rev. Cell Dev. Biol. 26, 137-156. doi: 10. 1146/annurev.cellbio.042308.113327

Zhang, H., Qu, X., Bao, C., Khurana, P., Wang, Q., Xie, Y., et al. (2010). Arabidopsis VILLIN5, an actin filament bundling and severing protein, is necessary for normal pollen tube growth. Plant Cell 22, 2749-2767. doi: 10.1105/tpc.110. 076257

Zhang, L., Xing, J., and Lin, J. (2019). At the intersection of exocytosis and endocytosis in plants. New Phytologist 224, 1479-1489. doi: 10.1111/nph.16018

Zhang, S., Liu, C., Wang, J., Ren, Z., Staiger, C. J., and Ren, H. (2016). A processive arabidopsis formin modulates actin filament dynamics in association with profilin. Mol. Plant 9, 900-910. doi: 10.1016/j.molp.2016.03.006

Zhang, X., Orlando, K., He, B., Xi, F., Zhang, J., Zajac, A., et al. (2008). Membrane association and functional regulation of Sec3 by phospholipids and Cdc42. J. Cell Biol. 180, 145-158. doi: 10.1083/jcb.200704128

Zhang, Y., and McCormick, S. (2010). The regulation of vesicle trafficking by small GTPases and phospholipids during pollen tube growth. Sex Plant Reprod. 23, 87-93. doi: 10.1007/s00497-009-0118-Z

Zheng, Y., Lin, X., Liang, H., Wang, F., and Chen, L. (2018). The long journey of pollen tube in the pistil. Int. J. Mol. Sci. 19:3529. doi: 10.3390/ijms19113529

Zhou, Y., Yang, Y., Niu, Y., Fan, T., Qian, D., Luo, C., et al. (2020). The tip-localized phosphatidylserine established by arabidopsis ala3 is crucial for rab gtpasemediated vesicle trafficking and pollen tube growth. Plant Cell 32, 3170-3187. doi: 10.1105/tpc. 19.00844

Zhou, Z., Shi, H., Chen, B., Zhang, R., Huang, S., and Fu, Y. (2015). Arabidopsis RIC1 severs actin filaments at the apex to regulate pollen tube growth. Plant Cell 27, 1140-1161. doi: 10.1105/tpc.114.135400

Zhu, J., Nan, Q., Qin, T., Qian, D., Mao, T., Yuan, S., et al. (2017). Higherordered actin structures remodeled by arabidopsis actin-depolymerizing factor 5 are important for pollen germination and pollen tube growth. Mol. Plant 10, 1065-1081. doi: 10.1016/j.molp.2017.06.001

Zhu, J., Wu, X., Yuan, S., Qian, D., Nan, Q., An, L., et al. (2014a). Annexin5 plays a vital role in Arabidopsis pollen development via $\mathrm{Ca}^{2+}$-dependent membrane trafficking. PLoS One 9:e102407. doi: 10.1371/journal.pone.0102407

Zhu, J., Yuan, S., Wei, G., Qian, D., Wu, X., Jia, H., et al. (2014b). Annexin5 is essential for pollen development in Arabidopsis. Mol. Plant 7, 751-754. doi: $10.1093 / \mathrm{mp} / \mathrm{sst} 171$

Zuo, X., Zhang, J., Zhang, Y., Hsu, S. C., Zhou, D., and Guo, W. (2006). Exo70 interacts with the Arp2/3 complex and regulates cell migration. Nat. Cell Biol. 8, 1383-1388. doi: $10.1038 /$ ncb1505

Conflict of Interest: The authors declare that the research was conducted in the absence of any commercial or financial relationships that could be construed as a potential conflict of interest.

Copyright (c) 2021 Ruan, Li, Wang and Ren. This is an open-access article distributed under the terms of the Creative Commons Attribution License (CC BY). The use, distribution or reproduction in other forums is permitted, provided the original author(s) and the copyright owner(s) are credited and that the original publication in this journal is cited, in accordance with accepted academic practice. No use, distribution or reproduction is permitted which does not comply with these terms. 